\title{
On the width of molecular states
}

\author{
M.Shmatikov \\ Russian Research Center "Kurchatov Institute", 123182 Moscow, Russia
}

\begin{abstract}
Width of molecular-type $Q \bar{Q} q \bar{q}$ hadronic states is discussed. It depends on the quantum numbers of the state and on the details of the long-range mechanism ensuring the bindedness of a molecule. Specific cases when the width is large are considered in detail.
\end{abstract}


Existence of multiquark exotic $Q Q \bar{q} \bar{q}$ states attracts interest for a long time since at present there are no evidence for any other states contaning more that 3 quarks which are stable with respect to strong decays. The possibility that such states could be bound for large enough $m_{Q} / m_{q}$ mass ratio was shown in [1, 2] to emerge as a consequence of flavor independence of chromoelectric forces. The interest to the problem was rekindled recently by the studies of of $Q Q \bar{q} \bar{q}$ states in the framework of chiral perturbation theory [3]. Qualitative conclusions of [3] can be formulated as follows: 1) in the infinite mass limit $\left(m_{q} \rightarrow \infty\right)$ stable $Q Q \bar{q} \bar{q}$ meson exists; 2) states containing $Q=c, b$ quarks may be weakly bound by the long-range one-pion forces which, moreover, are calculable in the chiral perturbation theory. The same conclusion as to existence of a bound state is valid also for a meson-antimeson $\bar{Q} q Q \bar{q}$ molecule.

It should be stressed that the infinite mass limit may be a poor approximation even for heavy $Q=c, b$ quarks. Then corrections $\simeq 1 / m_{Q}$ should be taken into account, the most important one being related to mass difference $\Delta m_{Q}$ between vector and pseudoscalar heavy mesons.

Investigation of molecular states consisting of heavy mesons with the account of finite heavy-quark corrections was carried out in [4, 5]. Onepion exchange (or, more precisely, its tensor component) was considered as a driving force for heavy-meson interaction. The effective coupling constant of the pion and the heavy meson $g$ was assumed to be universal for all heavy mesons due to pion interaction with the isovector current of a light quark only:

$$
\mathcal{L}_{i n t}=\frac{g}{f_{\pi}} \bar{q}(x) \gamma^{\mu} \gamma_{5} \vec{\tau} q(x) \partial_{\mu} \vec{\pi}(x)
$$

where $f_{\pi}$ is the $\pi$-meson decay constant $\left(f_{\pi} \approx 132 \mathrm{MeV}\right)$.

A number of deuteron-like loosely bound states was found in the charm and beauty sectors, the binding energy in the latter case amounting to about $50 \mathrm{MeV}$. In the channels with exotic flavor quantum numbers ( $Q Q \bar{q} \bar{q}$ states) pion exchange proves to be either too weak or even repulsive. The $H^{*} \bar{H}^{*}$ 
states (where $H^{*}$ is the generic notation for a vector $D^{*}$ or $B^{*}$ meson) are expected to decay into a pair of pseudoscalar $H \bar{H}$ mesons and it was conjectured in [5] that the corresponding width is about tens of $\mathrm{MeV}$.

In the present paper we investigate decay mechanisms of molecular-type heavy mesons with hidden flavor. First, such states can annihilate into ordinary mesons. One can get readily estimates for the annihilation width. Indeed, the radius of the annihilation $\left(\simeq 1 / m_{Q}\right)$ is much smaller than that of the bound state which can be assumed safely to be $\simeq 1 / \Lambda$, where $\Lambda$ is the QCD parameter. Then applying the familiar formula [6] we arrive immediately at

$$
\Gamma=\left(v \sigma_{\text {ann }}\right)\left|\psi_{\pi}(0)\right|^{2},
$$

where $v$ is the velocity of mesons bound in a molecular state, $\sigma_{a n n}$ is the annihilation cross section and $\psi_{\pi}$ is the wave function of the state generated by the one-pion-exchange force. For a loosely bound state the $\psi_{\pi}$ wave function can be approximated as follows

$$
\psi_{\pi}(r)=\sqrt{\frac{\kappa}{2 \pi}} \frac{\exp (-\kappa r)}{r}
$$

where $\kappa$ is the bound-state momentum related to the binding energy $\varepsilon$ : $\kappa=\sqrt{m_{Q} \varepsilon}$. The value of the $\left(v \sigma_{a n n}\right)$ can be extracted from the width of the pseudoscalar $Q \bar{Q}$ meson. For the positron-like system [7]

$$
\left(v \sigma_{a n n}\right) \approx \frac{32 \pi \alpha_{S}^{2}}{12 m_{Q}^{2}}
$$

Combining (3) and (4) we get

$$
\frac{\Gamma}{\varepsilon}=\sqrt{\frac{m_{Q}}{\varepsilon}} \cdot \frac{4}{3} \frac{\alpha_{S}^{2}}{\left(m_{Q} a\right)^{2}}
$$

where $a$ is the radius of the force. Taking the latter $a \approx 1 / 2 m_{\pi}$ [3]-[5] we rewrite (5) in the form

$$
\frac{\Gamma}{\varepsilon}=\sqrt{\frac{m_{Q}}{\varepsilon}} \frac{4}{3}\left(\alpha_{S} \frac{2 m_{\pi}}{m_{Q}}\right)^{2}
$$


yielding for the characteristic value of the binding energy $\varepsilon \approx 10 \mathrm{MeV}$ in the $D^{*} \bar{D}^{*}$ state

$$
\Gamma / \varepsilon \simeq 0.1
$$

For the $B^{*} \bar{B}^{*}$ state and/or nonzero orbital momenta this ratio will be even smaller.

Finite mass of a heavy quark makes vector $H^{*}$ mesons heavier than their pseudoscalar conterparts $H$. Nevertheless, for the apparent reason decay of $H^{*} \bar{H}$ and $H^{*} \bar{H}^{*}$ states with abnormal spin-parity quantum numbers into the $H \bar{H}$ pair is forbidden. These molecules will decay by annihilating into ordinary mesons with the width estimate (7) being applicable. At the same time bound states of heavy vector mesons $H^{*} \bar{H}^{*}$ with normal spin-parity quantum numbers can decay into a pair of pseudoscalar mesons: $H^{*} \bar{H}^{*} \rightarrow H \bar{H}$, and because of this decay channel their width may be large.

Let us consider the $H^{*} \bar{H}^{*}$ bound state with the quantum number $J^{\pi}=0^{+}$, involving two partial waves ${ }^{1} S_{0}$ and ${ }^{5} D_{0}$. Besides the $H^{*} \bar{H}^{*}$ state can couple to the $H \bar{H}$ pair with the $J^{\pi}=0^{+}$quantum numbers. Following 4 , 5 we bound our consideration by the most long range part of forces operating between mesons, i.e. one-pion exchange. Chiral invariance (at least approximately) determines coupling of the $\pi$-meson to a heavy meson. Note that for parity reasons the $H \bar{H} \pi$ vertex vanishes. One-pion exchange proves to be strong enough to bind a pair of vector mesons, the mass of the bound state $M$ being $2 m_{H}<M<2 m_{H^{*}} \llbracket 4$, 5]. Just the same mechanism (one-pion exchange) couples the bound state to the noninteracting $H \bar{H}$ pair. Thus we arrive at a system of 3 coupled channels

$$
H \bar{H}\left({ }^{1} S_{0}\right) \leftrightarrow H^{*} \bar{H}^{*}\left({ }^{1} S_{0}\right) \leftrightarrow H^{*} \bar{H}^{*}\left({ }^{5} D_{0}\right)
$$

Forbidding complexity of the problem necessitates making some simplifications. Having in mind the qualitative character of our results we neglect the contribution of the last channel in (8), since it provides some additional attraction only without changing the general character of interaction in the 
considered system. Then the potential matrix in the considered system reads

$$
V=\left(\begin{array}{cc}
0 & V_{12} \\
V_{21} & V_{22}
\end{array}\right)
$$

with $H \bar{H}$ and $H^{*} \bar{H}^{*}$ channels carrying numbers 1 and 2 respectively. Each component of the potential matrix can be represented in terms of one and the same function describing one-pion exchange

$$
V=-V_{0}\left(\vec{\tau}_{1} \cdot \vec{\tau}_{2}\right)\left[\begin{array}{cc}
0 & -\sqrt{3} \\
-\sqrt{3} & -2
\end{array}\right] \cdot \frac{\exp \left(-m_{\pi} r\right)}{m_{\pi} r},
$$

where $\vec{\tau}$ is the isospin Pauli matrix and $V_{0}$ is the "universal" coupling strength constant [4, 5]:

$$
V_{0}=\frac{m_{\pi}^{3}}{12 \pi} \frac{g^{2}}{f_{\pi}^{2}} .
$$

For the $g$ value as extracted from the $D^{*} \rightarrow D+\pi$ decay $(g \approx 0.6)$ this constant equals $V_{0} \approx 1.3 \mathrm{MeV}$. Note that one-pion exchange ensures attraction in the considered system in the isoscalar channel only (additional minus sign appears in the meson-antimeson case).

To take into account approximate value of coupling constants and investigate behavior of observables with their variation we modify interaction potential (9), (10) as follows

$$
\begin{aligned}
& V_{12}(r) \rightarrow V_{12}\left(\lambda^{\prime}, r\right)=\lambda^{\prime} \cdot V_{12}(r) \\
& V_{21}(r) \rightarrow V_{21}\left(\lambda^{\prime}, r\right)=\lambda^{\prime} \cdot V_{21}(r) \\
& V_{22}(r) \rightarrow V_{22}(\lambda, r)=\lambda \cdot V_{12}(r)
\end{aligned}
$$

where $\lambda$ and $\lambda^{\prime}$ are some numerical constants. The former constant controls the strength of interaction in the first $\left(H^{*} \bar{H}^{*}\right)$ channel and the latter the strength of its coupling to the "annihilation" $(H \bar{H})$ channel. The "reference" values of the potential strengths correspond then to $\lambda, \lambda^{\prime}=1$.

Interaction potentials $V_{22}$ and $V_{12}$ have comparable strength and equal range precluding application of perturbation theory approximations. Using 
the modified potential (19) and (12) we solve numerically the system of coupled Schrödinger equation and investigate behavior of the scattering length $\mathcal{A}$ in the second $\left(H^{*} \bar{H}^{*}\right)$ channel as the function of $\lambda$ and $\lambda^{\prime}$. In the case of uncoupled "annihilation" $(H \bar{H})$ channel (corresponding to $\left.\lambda^{\prime}=0\right)$ emergence of a (loosely) bound state is signalled by $\mathcal{A} \rightarrow \infty$ at some critical value of the strength coupling constant $\lambda_{c r}$. Coupling to the $H \bar{H}$ channel drastically changes behavior of $\mathcal{A}(\lambda)$. First, now molecule components spend some time in the $H \bar{H}$ state where interaction is absent. It implies that the average attraction in the considered system weakens. Then formation of a bound state requires corresponding increase of attraction in the $H^{*} \bar{H}^{*}$ state: domain of positive $\mathcal{A}$ 's, corresponding to bound states, shifts to the right along the $\lambda$-axis. For given value of $\lambda$ a bound state may even become an (unbound) virtual state. Besides, the $\mathcal{A}(\lambda)$ curve remains continuous for all values of $\lambda$. Second, the scattering length $\mathcal{A}$ acquires imaginary part. Its magnitude and behavior also depend on $\lambda$. The imaginary part $\operatorname{Im} \mathcal{A}$ achieves its extremum in the vicinity of the $\lambda$ value where a bound state emerges. Dependence of both real and imaginary parts of the $\mathcal{A}$ scattering length is illustrated in fig. 1 for the realistic case $\lambda^{\prime}=1$.

Inspection of curves in fig. 1 shows that the $\lambda$-behavior of the scattering length components reproduces the pattern of a resonance system with friction: absorption reaches its maximum just at the point where the real part of the resonance curve vanishes [8]. With weakening of "annihilation" strength ( $\lambda^{\prime}$ diminishing) the $\operatorname{Im} \mathcal{A}$ peak becomes more narrow and high. Hence the $\rho=\operatorname{Im} \mathcal{A} / \operatorname{Re} \mathcal{A}$ ratio may be both small and comparatively large $(\approx 1)$ depending on specific values of $\lambda$ and $\lambda^{\prime}$. It should be stressed that rapid variation of the $\rho$ ratio occurs in the region of "realistic" values $\left(\lambda, \lambda^{\prime}=1\right)$ and the extension of this region corresponds roughly to the accuracy with which the interaction potentials and hence $\lambda, \lambda^{\prime}$ strength coupling constants are known. These results are quite general and hold valid for any other (normal) spin-parity quantum numbers of the $H^{*} \bar{H}^{*}-H \bar{H}$ molecular states.

Resuming, we have considered the widths of molecular states consisting 
of heavy mesons. States involving vector and pseudoscalar $\left(H^{*} \bar{H}\right)$ and vector mesons $\left(H^{*} \bar{H}^{*}\right)$ with the abnormal spin-parity are expected to be very narrow decaying into ordinary mesons with the width given by $($ (]). Vector-meson $\left(H^{*} \bar{H}^{*}\right)$ molecular states with normal spin-parity quantum numbers will decay into the pair of pseudoscalar mesons $\left(H^{*} \bar{H}^{*} \rightarrow H \bar{H}\right)$. The qualitative conclusion is that the width of such states may vary in a wide range and, in particular, they can be rather broad and hence unobservable as resonances. The width of a resonance depends upon details of interaction mechanism and cannot be predicted with the state-of-the-art accuracy of coupling constants. We have considered the case when the mass of a molecular state exceeds the double mass of a heavy pseudoscalar meson $\left(M>2 m_{H}\right)$. Provided the binding energy is larger than the double mass difference between the vector and the pseudoscalar heavy meson $\left(M<2 m_{H}\right)$, the $H^{*} \bar{H}^{*} \rightarrow H \bar{H}$ decay channel will be closed and these molecular states will be stable with respect to strong decay. It should be noted, however, that for such strong binding characteristic momentum of the bound state is $\kappa_{H} \gtrsim \sqrt{m_{H} \cdot 2 \Delta m_{H}} \approx 700 \mathrm{MeV}$ and the corresponding characteristic distances $r_{H} \approx 1 / \kappa_{H} \approx 0.3 \mathrm{Fm}$. These values indicate that interacting heavy mesons are located well within the meson's quark-core domain and the calculations based on the analysis of long-range forces only may prove to be unreliable.

\section{References}

[1] J.-P.Ader, J.-M.Richard and P.Taxil, Phys.Rev. D25 (1982) 2370

[2] H.Lipkin, Phys.Lett. B172 (1986) 242

[3] A.V.Manohar and M.B.Wise, Nucl.Phys. B399 (1993) 17

[4] N.A.Törnqvist, Phys.Rev.Lett. 67 (1991) 556

[5] N.A.Törnqvist, Z.Phys. C61 (1994) 525 
[6] S.Deser et al., Phys.Rev. 96 (1954) 774

[7] L.D.Landau and E.M.Lifshitz, Relativistic quantum mechanics, Pergamon Press, 1978

[8] L.D.Landau and E.M.Lifshitz, Mechanics, Pergamon Press, 1976 


\section{Figure caption}

Fig.1. Dependence of the scattering length $\mathcal{A}[\mathrm{Fm}]$ in the $H^{*} \bar{H}^{*}$ channel upon the relative strength constant $\lambda$. Solid curves represent behavior of the real and imaginary parts of $\mathcal{A}$ for $\lambda^{\prime}=1$ (full-fledged coupling to the "annihilation" $H \bar{H}$ channel), while dotted curve is for the real part of $\mathcal{A}$ for $\lambda^{\prime}=0$ (uncoupled "annihilation" channel: imaginary part of $\mathcal{A}$ vanishes). "Reference" values of relative strength coupling constants are $\lambda, \lambda^{\prime}=1$. 
This figure "fig1-1.png" is available in "png" format from: http://arxiv.org/ps/hep-ph/9503471v1 